\title{
Misdiagnosis \\ Cardiac Metastasis Presented as a Pseudo-Infarction on Electrocardiography
}

\author{
Kuo Li PAN, ${ }^{1}$ MD, Lung Sheng WU, ${ }^{2}$ MD, Chang Min ChunG, ${ }^{1}$ MD, \\ Shih Tai CHANG, ${ }^{1} \mathrm{MD}$, Pi Chi Lin, ${ }^{1} \mathrm{MD}$, and Jen Te Hsu, ${ }^{1} \mathrm{MD}$
}

\begin{abstract}
SUMMARY
Most tumor invasion into the heart is nonspecific and clinically silent. Myocardium metastasis rarely mimics a myocardial infarction. In this case, a cardiac metastasis from a squamous cell carcinoma presented with both persistent ST elevation and paroxysmal supraventricular tachycardia. The secondary lesion was located in the anterior wall and lateral wall of the left ventricle and induced electrocardiographic changes imitating an acute myocardial infarction. (Int Heart J 2007; 48: 399-405)
\end{abstract}

Key words: Pseudo-infarction, PSVT, Cardiac metastasis, Echocardiography, Squamous cell carcinoma

$\mathrm{S}_{\mathrm{T}}$ elevation is most commonly due to coronary occlusion with underlying atherosclerosis and it is less commonly due to coronary artery spasms. Metastasis disease has been identified as a cause of coronary artery occlusion and a rare cause of acute myocardial infarction. ${ }^{1,2)}$ However, ST elevation can also be secondary to tumor invasion of the myocardium. ${ }^{3-5)}$ This study describes a patient with cardiac metastasis from a squamous cell lung cancer who presented with a prolonged ST segment elevation suggestive of myocardial infarction.

\section{CASE REPORT}

A 73-year-old female was admitted to an emergency department with progressive fatigue and obtundation. The patient's clinical history was unremarkable, except for a compression fracture of the $8^{\text {th }}$ vertebral body and a backache prior to admission.

Although physical examination determined that the patient was ill and disoriented in terms of time and space, no focal neurological deficits were found.

From the ${ }^{1}$ Division of Cardiology, Chiayi Chang Gung Memorial Hospital, Chang Gung University College of Medicine, ${ }^{2}$ First Division, Cardiovascular Section, Lin-Kou Medical Center, Chang-Gung Memorial Hospital, Taipei, Taiwan.

Address for correspondence: Jen Te Hsu, MD, Division of Cardiology, Chiayi Chang Gung Memorial Hospital, 6, Sec. West Chai-Pu Road, Pu-Tz City, Chai Yi Hsien, Taipei, Taiwan.

Received for publication October 27, 2006.

Revised and accepted Febrary 15, 2007. 


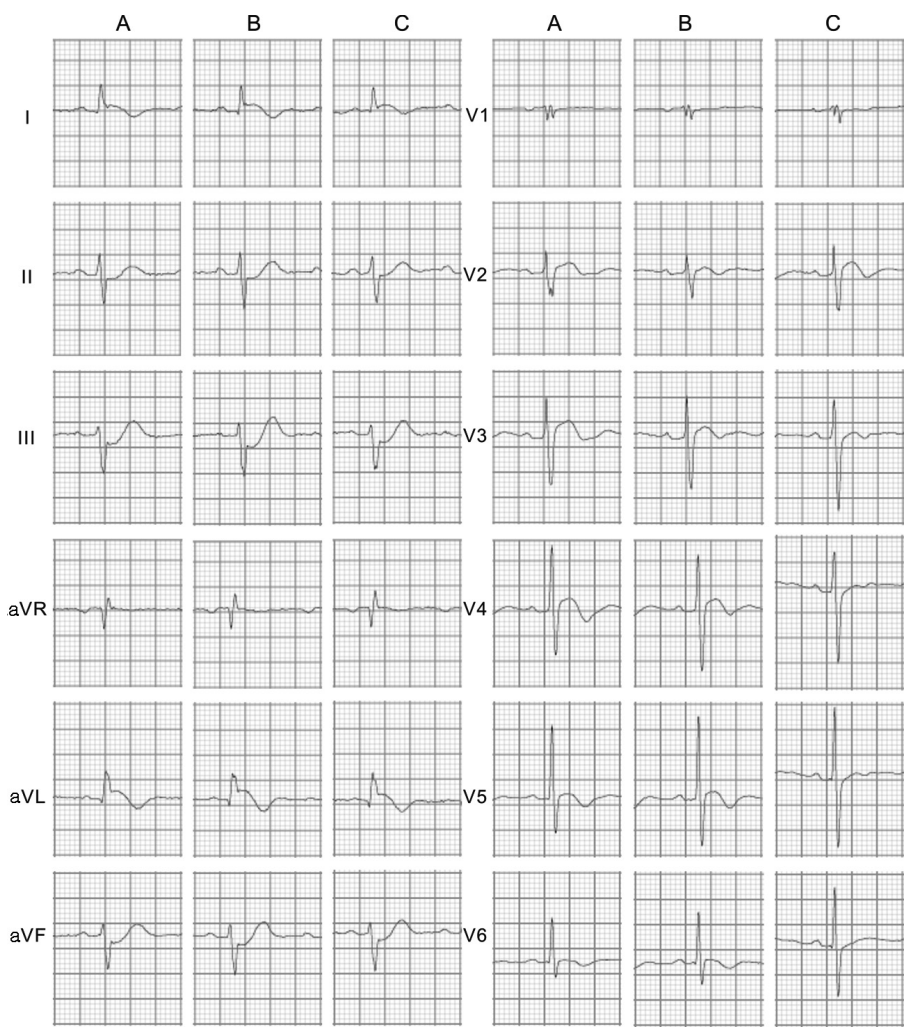

Figure 1. Electrocardiogram demonstrated persistent marked S-T segment elevation and $\mathrm{T}$ inversion in leads $\mathrm{I}, \mathrm{aVL}, \mathrm{V}_{2}-\mathrm{V}_{5}$, in the absence of a $\mathrm{Q}$ wave. Serial electrocardiograms for (A) day 1 (B), day $3(\mathbf{C})$, and day 14 are shown.

She was rated 12/15 according to the Glasgow Coma Scale. Her blood pressure was $107 / 63 \mathrm{mmHg}$ and the pulse rate was $87 \mathrm{beat} / \mathrm{min}$. She was neither tachypneic nor febrile. Her conjunctiva was slightly pale. On auscultation, her breathing was clear and diminished heart sound without any murmur was detected. Blood biochemistry revealed hypercalcemia. The adjusted plasma calcium was $12.1 \mathrm{mg} /$ dL (normal range: 7.9-9.9 mg/dL). Chest radiography demonstrated cardiomegaly and increased soft tissue density in the left lower lung with obliteration of the left heart border.

An electrocardiogram revealed marked elevation of the ST segment and $\mathrm{T}$ inversion in leads $\mathrm{I}, \mathrm{aVL}$, and $\mathrm{V}_{2}-\mathrm{V}_{5}$ in the absence of a $\mathrm{Q}$ wave (Figure 1A). Reciprocal change of the ST depression was also noted at leads II, III, and aVF. The ECG findings were compatible with anterior and lateral wall myocardial infarction. This patient did not complain of chest tightness or chest pain at the time 
electrocardiographic abnormalities were identified.

The patient was admitted to the coronary care unit under a diagnosis of acute myocardial infarction and hypercalcemia. She was administered heparin and tirofiban immediately. Prior to further coronary angiography, a transthoracic echocardiogram revealed a huge echogenic mass located at the anterior and lateral aspect of the left ventricle (LV), with myocardium invasion, accompanied by moderate pericardial effusion (Figure 2). Moreover, echocardiography also showed hypokinesis where the tumor invaded the myocardial walls. The serial creatine kinase MB and cardiac troponin I were within normal limits. The cardiac troponin levels were $0.13 \mathrm{ng} / \mathrm{mL}$ (cut-off value for acute coronary syndrome: $0.16 \mathrm{ng} / \mathrm{mL}$ ) at one hour. The follow-up cardiac troponin level was $0.14 \mathrm{ng} / \mathrm{mL}$ at 6 hours.

Coronary angiography was avoided due to the condition of the patient, echocardiography findings, and normal cardiac isoenzyme levels.

Complete blood count, serial cardiac enzyme studies, and levels of creatine phosphokinase and creatine kinase MB isoenzyme and aspartate aminotransferase were checked and within normal limits. Additionally, the intact parathyroid hormone (iPTH) level was $<3.0 \mathrm{pg} / \mathrm{mL}$ (normal range, $7-53 \mathrm{pg} / \mathrm{mL}$ ). Initial

A

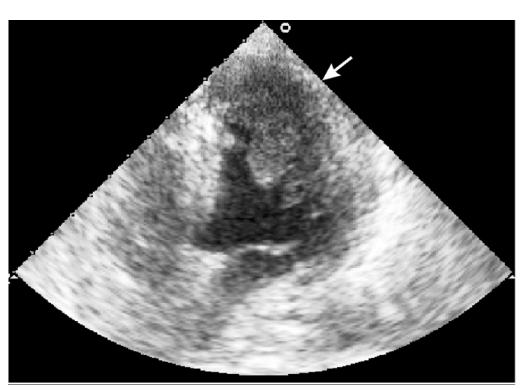

C

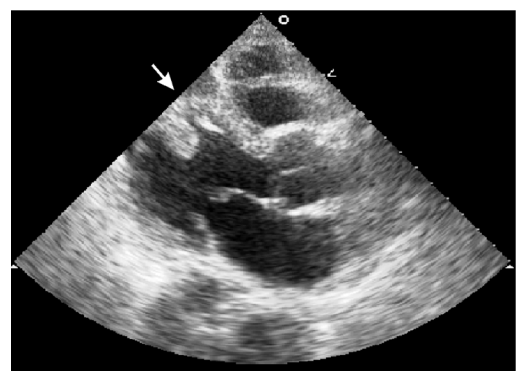

B

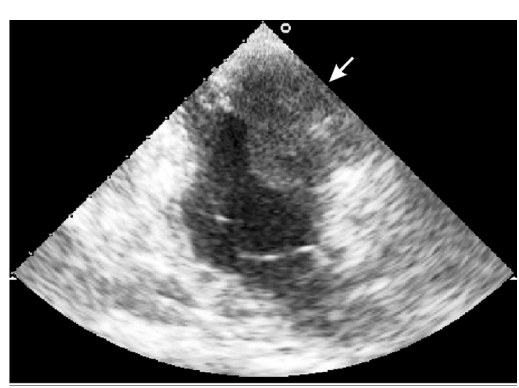

D

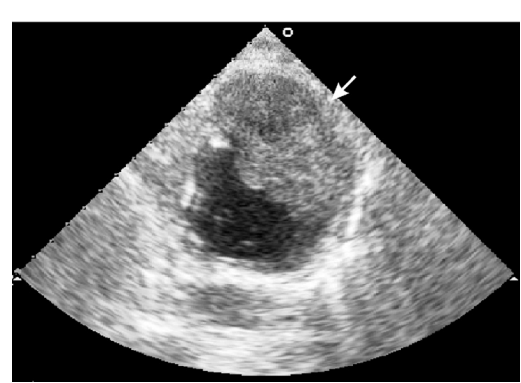

Figure 2. Transthoracic apical 4-chamber (A), apical 2-chamber (B), parasternal long axis (C), and parasternal shortaxis (D) views of the heart, showing a large tumor mass that invaded the anterolateral myocardium and pericardium (arrows). 


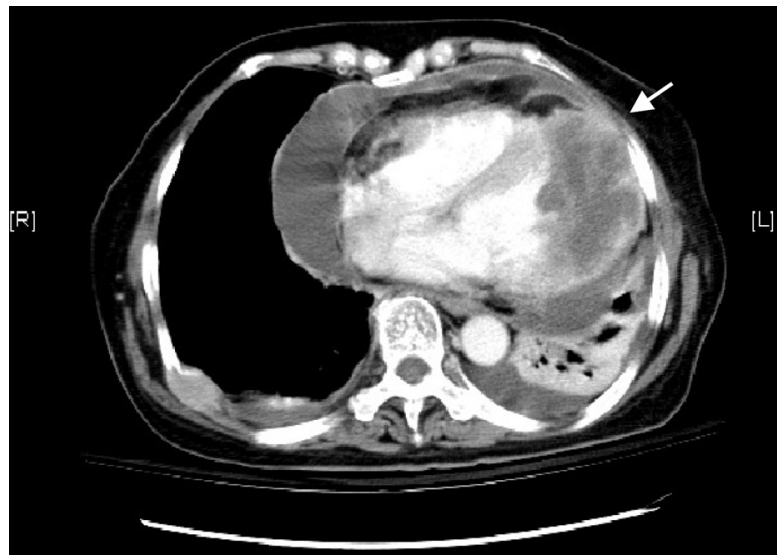

Figure 3. Computed tomographic findings of the chest showing a heterogeneous mass invading the lateral wall (arrow) of the left ventricle with large pericardial effusion.

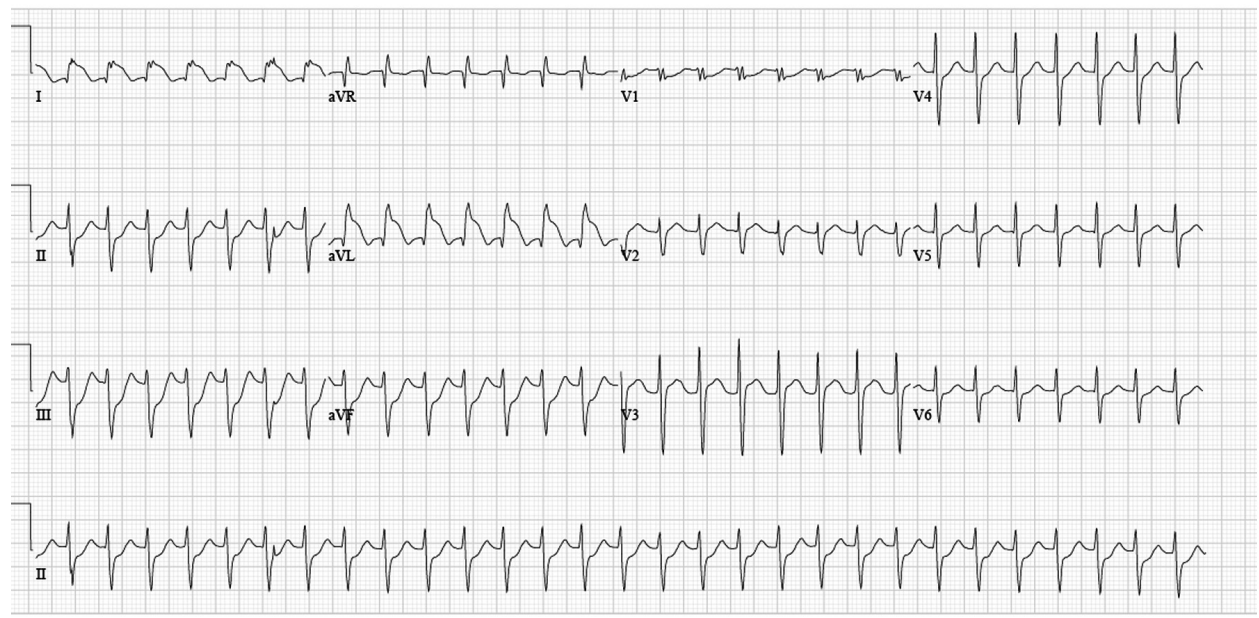

Figure 4. Electrocardiogram on hospitalization day 14 that showed paroxysmal supraventricular tachycardia.

antiplatelet and anticoagulation therapy was withdrawn after the above studies.

Her level of consciousness returned gradually after hydration with normal saline and reached a Glasgow Coma Scale score of 15/15. She was transferred from the coronary care unit to an ordinary ward after the adjusted plasma calcium was reduced to $10.1 \mathrm{mg} / \mathrm{dL}$.

Unfortunately, a pathologic right femur fracture occurred and she accepted open reduction. Bony erosion by a tumor was incidentally noted during surgery. 
Pathologic sections demonstrated metastatic squamous cell carcinoma.

Computed tomography (CT) of the head, chest, abdomen, and pelvis revealed a $8.5 \times 6 \times 5 \mathrm{~cm}$ heterogeneous enhanced extensive transmural tumor in the left pericardium with LV invasion and large pericardial effusion (Figure 3). Furthermore, multiple metastasis tumors were also identified in the right hilum, left lateral chest wall, periaortic space, right anterior abdominal wall, left psoas muscle, and bilateral adrenal glands. Multiple bone metastases were also identified.

Serial ECG was unchanged during 2 weeks of hospitalization (Figure 1B, C). The patient had 1 paroxysmal supraventricular tachycardia (PSVT) attack on day 14 (Figure 4). This arrhythmia recovered spontaneously. No invasive electrophysiological study was performed due to her poor condition.

Although the origin of the primary tumor was not clearly demonstrated by $\mathrm{CT}$, this patient was treated as having lung cancer and she agreed to undergo chemotherapy. On the second admission, her clinical condition deteriorated due to severe urosepsis and she died 2 months later.

\section{Discussion}

The most common neoplasms associated with cardiac metastasis are lung cancer, esophageal cancer, lymphoma, breast cancer, leukemia, stomach cancer, and melanoma. ${ }^{6,7)}$ An autopsy series determined that the incidence of cardiac metastasis in patients with malignant disease was as high as $22-31 \% .^{8-10)}$ Cardiac metastases, however, are difficult to diagnose before death, because there are frequently few typical signs that are indicative of cardiac metastasis and these signs are often clinically unapparent.

This report describes a patient with an ECG suggestive of anterior and lateral myocardial infarction. This case differed from previous reports in two principal ways.

First, this patient experienced reduced consciousness related to hypercalcemia and did not present with any chest discomfort. Abnormal ECG findings were the first clue that led to a diagnosis of cardiac metastasis. Hypercalcemia was due to a terminal presentation of cancer metastasis. The low iPTH was secondary to hypercalcemia that was related to multiple bony metastases. Although some reports have also described asymptomatic ECG change with cardiac metastasis, none of the patients were complicated with a change of consciousness.

Second, the serial ECG demonstrated both persistent ST elevation and an episode of PSVT. Notably, the ECG revealed persistent ST elevation without development of evolutionary ECG changes and Q wave formation. These findings differed from ECG findings characteristic of myocardial infarction. The 
ECG findings were compatible with the description by Hartman, et al. ${ }^{3)}$

Arrhythmia has been frequently found in patients with cardiac metastasis, especially in patients with myocardial involvement. Various arrhythmias were described by two retrospective autopsy studies. Cates, et al explored various supraventricular arrhythmias including atrial fibrillation, atrial flutter, and paroxysmal atrial tachycardia. ${ }^{8)}$ Tamura, et al reported a high incidence (35\%) of concurrent supraventricular arrhythmia including PSVT (8\%) in their series. ${ }^{10)}$ Although PSVT was not proved to be related to myocardial invasion in this case, it was reasonably suspected in this elderly patient. Only Yao, et al reported a similar case that presented with both ST-elevation and PSVT. ${ }^{11)}$

In patients with cardiac metastasis, the pericardium is the most frequent site of involvement, and patients typically present with clinical symptoms and signs of acute pericarditis. ${ }^{10)}$ In some cases, the patient presents with cardiac tamponade. ${ }^{2)}$ Other presentations are arrhythmia and myocardial infarction. The myocardium accounts for only approximately $12 \%$ of cardiac metastasis in autopsy lung cancer cases. ${ }^{12)}$ Such cardiac metastasis is rarely associated with ST segment elevation. In this patient, ECG changes were the primary clue of myocardial invasion.

Elevation of the ST segment secondary to a neoplastic process can be due to a primary heart tumor ${ }^{13)}$ or metastasis tumor invasion into the myocardium, ${ }^{4,5}$ tumor emboli within a coronary artery, ${ }^{14)}$ or a metastatic lesion surrounding a coronary artery. ${ }^{1,2)}$

This patient did not have enzyme and ECG evolution characteristic of acute myocardial infarction. The ST segment elevation was due to a metastatic tumor that invaded the pericardium and myocardium.

Suggested mechanisms for these pseudo-infarction ECG patterns are continuous myocardial injury preventing formation of new cardiac cell membrane, stretched adjacent muscle fibers, inflammatory reaction, change in the transmembrane $\mathrm{Na}^{+}-\mathrm{K}^{+}$gradient, and transfer of $\mathrm{K}^{+}$from damaged tissue to the adjacent myocardium producing electropotential differences. ${ }^{15-16)}$

Conclusion: In summary, this report has described a patient with metastasis cancer of the myocardium that was misdiagnosed as acute myocardial infarction. However, the clinical presentation, serial cardiac enzyme levels, and prolonged ST elevation findings all ruled against such a diagnosis.

When patients have ECG changes suggestive of myocardial infarction or ischemia, echocardiography is critical for confirming this diagnosis, especially in patients with atypical presentations which include reduced consciousness and no chest discomfort. Metastatic cancer should be included in differential diagnosis for patients with persistent ST elevation and unexplained hypercalcemia, especially in patients with known or suspected malignancies. 
Furthermore, although myocardial infarction due to malignancy is a rare condition, coronary catheterization was suggested to differentiate real coronary occlusion from pseudo-infarction ECG patterns in patients with elevated cardiac enzymes.

\section{REFERENCES}

1. Franciosa JA, Lawrinson W. Coronary artery occlusion due to neoplasm. A rare cause of acute myocardial infarction. Arch Intern Med 1971; 128: 797-801.

2. Cook MA, Sanchez EJ, Lopez JJ Jr, Bloomfield DA. Acute myocardial infarction: a rare presentation of pancreatic carcinoma. J Clin Gastroenterol 1999; 28: 271-2.

3. Hartman RB, Clark PI, Schulamn P. Pronounced and prolonged ST segment elevation: a pathognomonic sign of tumor invasion of the heart. Arch Intern Med 1982; 142: 1917-9.

4. Rodrigues AC, Abreu E, Demarchi LM, Mathias W Jr, Leal SM, Andrade JL. Lung neoplasm mimicking an acute lateral myocardial infarction. J Am Soc Echocardiogr 2003; 16: 1198-200.

5. Astorri E, Bonetti A, Fiorina P. ECG mimicking acute myocardial infarction during heart involvement by lung neoplasm. Int J Cardiol 2000; 74: 225-6.

6. Lam KY, Dickens P, Chan AC. Tumors of the heart. A 20-year experience with a review of 12,485 consecutive autopsies. Arch Pathol Lab Med 1993; 117: 1027-31.

7. Abraham KP, Reddy V, Gattuso P. Neoplasms metastatic to the heart: review of 3314 consecutive autopsies. Am J Cardiovasc Pathol 1990; 3: 195-8.

8. Cates CU, Virmani R, Vaughn WK, Robertson RM. Electrocardiographic markers of cardiac metastasis. Am Heart J 1986; 112: 1297-303.

9. Strauss BL, Matthews MJ, Cohen MH, Simon R, Tejada F. Cardiac metastases in lung cancer. Chest 1977; 71: 607-11.

10. Tamura A, Matsubara O, Yoshimura N, Kasuga T, Akagawa S, Aoki N. Cardiac metastasis of lung cancer. A study of metastatic pathways and clinical manifestations. Cancer 1992; 70: 437-42.

11. Yao NS, Hsu YM, Liu JM, Chen LT, Liau CS. Lung cancer mimicking acute myocardial infarction on electrocardiogram. Am J Emerg Med 1999; 17: 86-8.

12. Abe S, Watanabe N, Ogura S, et al. Myocardial metastasis from primary lung cancer: myocardial infarctionlike ECG changes and pathologic findings. Jpn J Med 1991; 30: 213-8.

13. Lehrman KL, Prozan GB, Ullyot D. Atrial myxoma presenting as acute myocardial infarction. Am Heart $\mathbf{J}$ 1985; 110: 1293-5.

14. Aragon J. A rare noncardiac cause for acute myocardial infarction in a 13-year-old patient. Cardiol Rev 2004; 12: 31-6.

15. Nakamura A, Suchi T, Mizuno Y. The effect of malignant neoplasms on the heart. A study on the electrocardiographic abnormalities and the anatomical findings in cases with and without cardiac involvement. Jpn Circ J 1975; 39: 531-42.

16. Harris TR, Copeland GD, Brody DA. Progressive injury current with metastatic tumor of the heart. Am Heart J 1965; 69: 392-400. 